\title{
The Research of MES Material Management Base on BOM
}

\author{
Fan Guoliang* , Zhang Guangshen, Xu Qilin \\ Information and Software Development Center, Beijing Institute of Aerospace Control Devices, Beijing, China
}

\section{Email address:}

fgliang_bj@163.com (Fan Guoliang)

${ }^{*}$ Corresponding author

\section{To cite this article:}

Fan Guoliang, Zhang Guangshen, Xu Qilin. The Research of MES Material Management Base on BOM. Science Discovery. Vol. 9, No. 2, 2021, pp. 74-78. doi: 10.11648/j.sd.20210902.19

Received: March 17, 2021; Accepted: April 22, 2021; Published: April 26, 2021

\begin{abstract}
MES is an information system for workshop between the planning system and operation control system. It is the basic technical means to realize the automation and agility of production management. The functions of MES include resource allocation, process management, quality control, material management, etc. MES can achieve the function of information tracking, information analysis. It can also predict what may happen in the production process. BOM can describe the material composition of a product and the relationship between its finished materials. MBOM contains the detailed information of various parts, such as the source, quantity and the difference of using time. In essence, it is the description of product materials. Product materials are the core resource of manufacture and management. MBOM provide important information of inventory preparation and material distribution. The traditional material management mode only focuses on the business of purchase, distribution and storage. The relationship between various materials is not considered. Based on the study of the traditional material management mode, this paper puts forward the MES material management mode based on BOM. The information management system is designed and developed according to this management mode. Through the verification by practical application, the material management mode based on BOM realizes the reasonable allocation of materials. Using BOM can improve the efficiency of MES material management.
\end{abstract}

Keywords: MES, MBOM, Material Management

\section{基于BOM的制造执行系统物料管理方法研究}

范郭亮”，张广申，徐其林

信息化与软件开发中心, 北京航天控制仪器研究所, 北京, 中国

邮箱

fgliang_bj@163.com（范郭亮）

摘要: 制造执行系统是面向车间现场的管理系统, 处于企业计划层和车间层操作控制系统之间, 是实现生产管理自动 化与敏捷化的基本技术手段。MES的功能包括车间的资源配置、过程管理、质量控制、物料管理等，实现对生产信息 跟踪、信息分析, 并对可能发生的事情进行预测。BOM数据可以描述产品的物料组成及其产成品物料之间的关系。制 造BOM中包含了各类零件的详细信息, 包括物料的来源、物料的使用数量、物料使用的时间差异等。其本质上是对产 品制造物料数据的描述。产品制造物料数据是制造企业生产组织和管理的核心资源。MBOM可以为MES的库存准备及 物料配送提供重要信息。将制造BOM应用于MES物料管理, 能够提高物料出入库、库存管理的执行效率。

关键词：制造执行系统，制造BOM，物料管理 


\section{1. 引言}

数字化企业是1996年以后出现的一个概念, 是现代企 业运营的一种新模式[1]。20世纪90年代以来，信息技术的 发展极为迅速, 经济全球化的趋势日益加剧, 从而促成了 市场环境的根本性变化。面对激烈竞争, 企业界的重要对 策之一是技术和管理的高度融合, 即将制造技术与信息技 术、自动化技术、现代管理技术和系统科学技术有机融合 起来, 形成新一代先进制造技术, 以此来支撑新的业务模 式的转变。为此, 企业资源计划 (ERP) 和制造执行系统 (MES) 从理念发展到软件平台, 再形成完整的系统, 并 逐步开始了应用和普及的过程 [2,3]。

制造执行系统 (Manufacturing Execution System, MES) 是面向车间现场的管理系统，处于企业计划层和车间层 操作控制系统之间的执行层。MES不仅能实现对生产信息 的跟踪, 而且能对信息加以分析, 并对可能发生的事情进 行预测 $[4,5]$ 。

MES的任务是根据上级下达的生产计划, 充分利用车 间的各种生产资源、生产方法和丰富的实时现场信息，快 速、低成本地制造出高质量的产品。

其生产活动涉及订单管理、设备管理、库存跟踪、物 料流动、数据采集, 以及维护管理、质量控制、性能分析 及人力资源管理等 $[6,7]$ 。

MES汇集了车间中用以管理和优化从下定单到产成 品的生产活动全过程的相关硬件或软件构件, 它控制和利 用实时准确的制造信息来指导、响应并报告车间发生的各 项活动, 同时向企业决策支持过程提供有关生产活动的任 务评价信息。MES的功能包括车间的资源分配、过程管理、 质量控制、维护管理、数据采集、性能分析及物料管理等。 由此可见, MES是车间信息集成、实现生产管理自动化与 敏捷化的基本技术手段。作为车间层的先进生产管理技术， MES系统的集成性、柔性、开放性、自组织、自适应性对 车间制造过程的优化运行和敏捷性发挥着重要作用 $[8,9]$ 。

本文针对MES物料管理功能进行了研究。传统物料管 理模式只关注物料的进、出、存等业务管理, 没有考虑各 物料之间的联系。实际生产过程中,各物料的数量必须进 行合理的配置, 才能提升产品生产效率。BOM数据可以描 述产品的物料组成及其产成品物料之间的关系。将BOM 数据模型引入到MES物料管理, 能够实现MES物料的合理 配置, 优化库存准备及物料配送方案, 并提升物料出入库、 库存管理的执行效率。

\section{BOM与物料管理}

BOM（Bill of Material）数据既用来描述产品的物料 组成及其产成品的物料之间关系, 也用来表示产品的说明 书、产品包装、零部件的相关文档、质量标准等其他信息。 在制造业中, BOM 是核心的基础数据, 是产品数字化定 义的重要内容, 是连接产品工程设计和生产经营管理的桥 梁。BOM表达的是产品的结构或装配视图, 是一种以产 品为中心的建模方法 [10]。
$\mathrm{BOM}$ 是工厂重要的产品信息载体, 完成各部门的信 息传递与共享，贯穿于产品设计、工艺设计和产品制造、 产品交付和售后服务的全过程。BOM主要包含设计BOM

(Engineering BOM, EBOM)、工艺BOM (Process BOM, $\mathrm{PBOM}$ )、制造BOM（Manufacturing BOM, MBOM）、 生产BOM（Order BOM, OBOM）、交付BOM（Delivery BOM，DBOM）、客服服务BOM ( Service BOM，SBOM) 等。

制造过程是一个由原材料、毛坏、外购件等逐步变成 在制品、半成品、成品的过程。在生产过程的每一个步骤 中，都要涉及一定的资源、设备、人员等，另外还要有与 之相关的基础数据, 如设计数据、工艺数据、库存数据、 装配数据等。通常, 这些数据都包含在BOM中。

$\mathrm{MBOM}$ 管理从本质上是对产品制造物料数据的管理。 产品制造物料数据是指产品在研发、制造、售后服务等全 生命周期不同阶段产生及应用的所有数据, 是制造企业生 产组织和管理的核心资源 $[11,12]$ 。

MBOM中包含了各类零件的详细信息, 如物料的来源、 物料的使用数量、物料使用的时间差异等, 是MRP不可缺 少的基础数据, 同时也为MES中的库存准备及物料配送提 供了重要数据。由于明确了物料的使用时间、数量、类型 和来源, 库房可以清晰明了的查看各个工位所需物料的数 量及时间, 当任务下达后, 可根据物料需求计划及时安排 物料的采购及调运; 同时协同过程管理和产品跟踪系统所 提供的数据, 能获取现场的物料使用情况及物料的当前需 求量, 通过这些信息可及时进行物料的准备及配送, 并对 现有库存余量进行监控, 及时调整外协件的供应, 确保生 产的正常运行 $[13,14]$ 。

\section{3. 详细解决方案}

制造BOM中包含库存管理的相关信息, 将制造BOM 模型应用于MES物料管理, 可以提高物料管理效率, 增强 物料模块可用性。

图1是MES物料管理功能框图。

物料主数据管理系统提供物料基础数据, 包括: 物料 编码以及物料名称、图号、规格型号、物理状态属性等。 物料主数据是构建 $\mathrm{BOM}$ 的基础, 也为物料流动、查询、 追溯提供了数据支撑 $[15,16]$ 。

物资备料完成触发物料管理模块领料入库流程, 同时 MES物料管理模块根据生产计划及库存, 产生物料需求信 息并反馈给物资备料。

物料管理中借助制造BOM数据, 提高基础数据检索、 库存查询速度, 也可将物料信息以树状结构清晰明了地进 行展示[17,18]。 


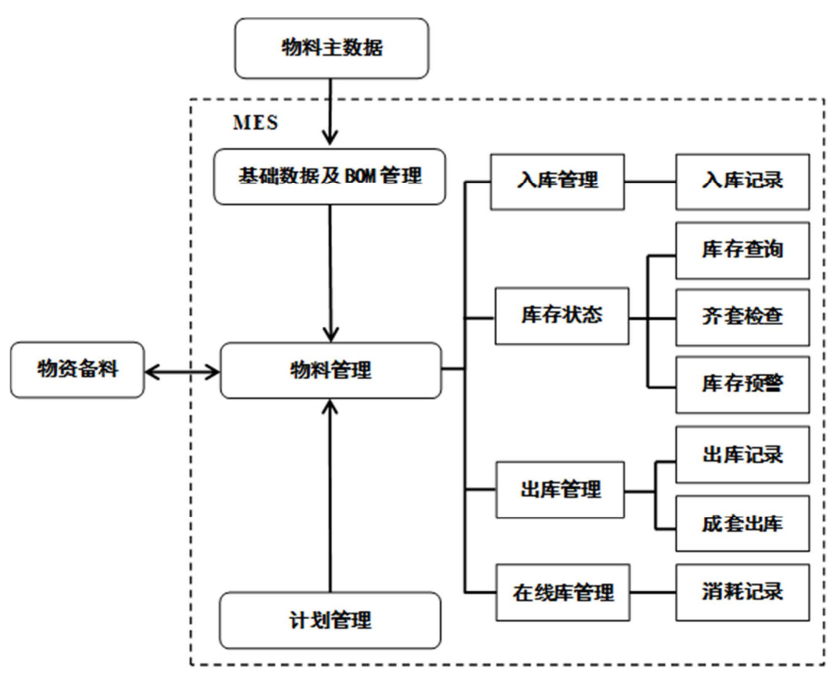

图1 MES物料管理功能图。

\section{1. 入库管理}

图 2 是入库流程, 数量、批次等采购信息及合格证等 质量信息与物资备料过程有关，但对于多批次、重复备料 的物料而言, 物料编码、规格型号等基础数据是保持不变 的。在创建入库单时, 可以将物料基础信息以 $\mathrm{BOM}$ 树状 结构显示, 通过点选获取物料基础信息, 快速完成入库单 创建。

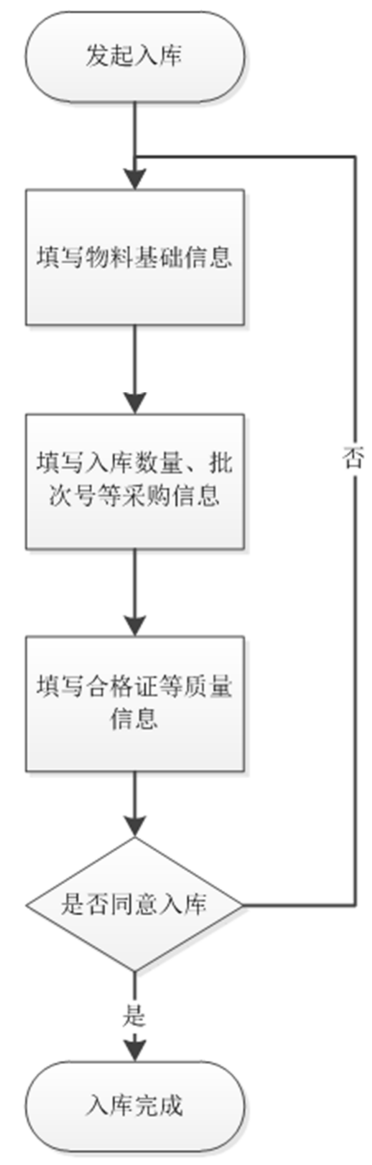

图2 入库流程图。

\section{2. 库存状态}

\subsection{1. 库存查询}

传统的查询页面使用输入框获取查询条件, 若采用 3 图所示的BOM树状结构, 并通过点选输入查询条件, 可 以简化库存查询过程, 并使库存信息以产品结构或装配视 图形式展现, 方便库房管理人员直观掌握库存情况。

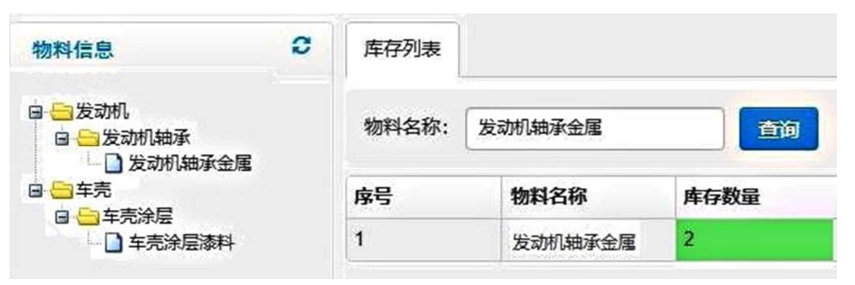

图3 库存查询。

\subsection{2. 齐套检查}

当制定生产计划后, 库房管理人员需要完成库房齐套 检查, 以了解原材料、元器件、标准件、外购件、半成品 等物料的库存是否满足生产计划。引入 较为便捷地实现齐套性检查。假设生产计划套数为 $\mathrm{P}$, BOM中某物料单套用量为 $\mathrm{n}$, 该物料库存数量为 $\mathrm{N}$, 建立 齐套检查模型如（1）（2）:

$$
\begin{gathered}
D=P \times n-N \\
\left\{\begin{array}{l}
D \geq 0 \quad \text { 满足 } \\
D<0 \quad \text { 不满足 }
\end{array}\right.
\end{gathered}
$$

齐套检查示意图如图4所示, 绿色表示满足齐套, 红 色表示不满足齐套，使齐套性检查显示更加直观。

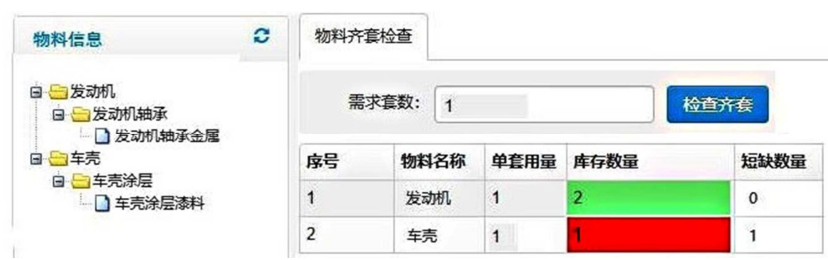

图4 齐套检查。

\section{3. 出库管理}

\subsection{1. 出库记录}

图5 是出库流程图。BOM直观展示生产所需的原材料、 元器件、标准件、外购件、半成品等物料及其结构、生产 装配顺序等信息。 


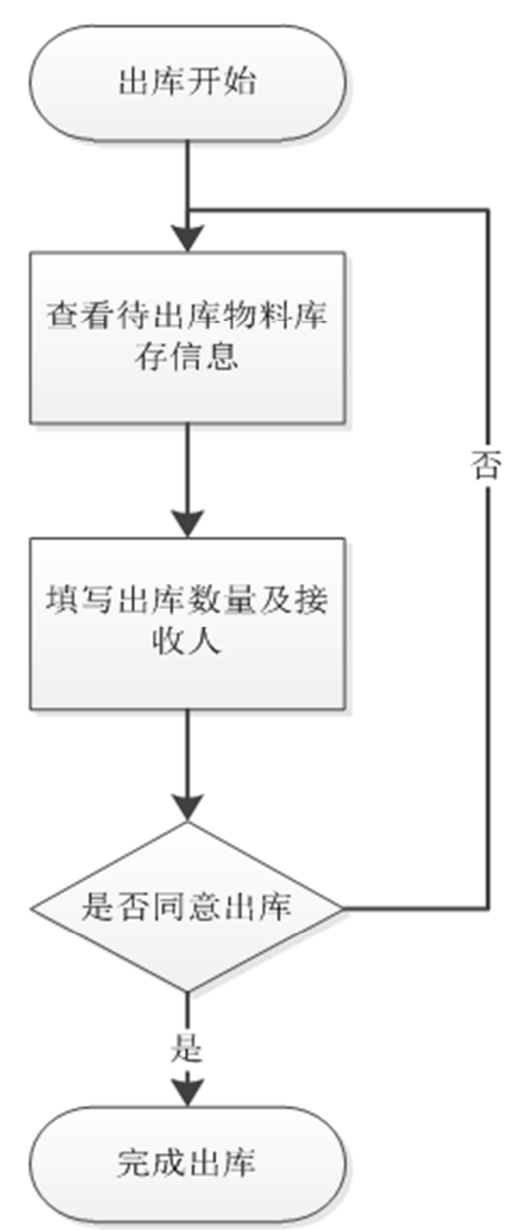

图5 出库流程图。

利用BOM树可以快速获得待出库物料的库存信息如 图6所示。

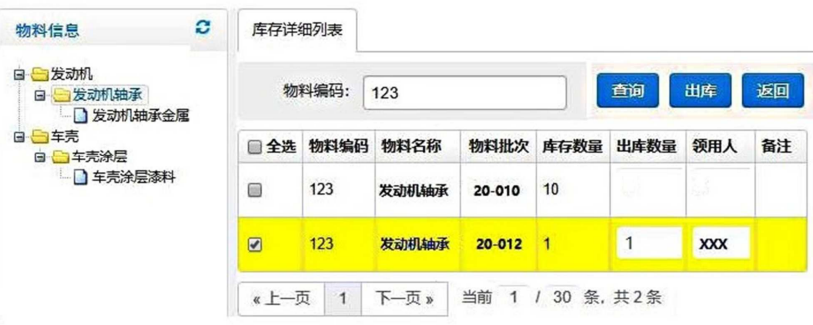

图6 物料出库。

\subsection{2. 成套出库}

成套出库是根据BOM信息快速完成某种产成品或半 成品生产所需的全部物料齐套检查及出库。假设 $\mathrm{S}$ 是BOM 中的所有物料集合, 第 $\mathrm{i}$ 种物料为 $\mathrm{x}_{\mathrm{i}}$, 其单套用量为 $\mathrm{n}_{\mathrm{i}}$, 生 产计划套数为 $\mathrm{P}$, 该物料库存数量为 $\mathrm{N}, \mathrm{BOM}$ 树中共有物 料 $\mathrm{m}$ 项，建立齐套检查模型如（3）:

$$
\bigcup_{i=1}^{m}\left(x_{i} \mid x_{i} \in S, P \times n_{i}-N \geq 0\right)
$$

\section{4. 结论}

$\mathrm{MES}$ 是生产制造企业实现生产管理自动化与敏捷化 的基本技术手段。制造BOM中包含库存管理及配送信息。 将BOM树应用于MES系统物料管理, 能够使物料出入库、 库存管理等功能更加贴近业务需求, 使相关功能操作更加 简便灵活, 有利于提高生产部门物料管理科学性、合理性。

本文在对传统物料管理方式研究的基础上, 提出基于 BOM的MES物料管理模式, 并依据该管理模式设计开发 了MES系统。通过实际应用检验，基于 BOM的MES物料 管理模式实现了物料的合理配置, 提升了物料出入库及库 存管理的执行效率。

\section{参考文献}

[1] 亚德里安.J.斯莱沃斯基. 数字化企业[M].北京:中信出版 社,2001.

[2] 何船. 基于MES的数字化工厂构建 [J]. 机械设计与制造工程, 2019, 48(2):77-81.

[3] 王军,李想,张萌等.基于MES的汽车空调活塞自动化生产线 设计 [J].组合机床与自动化加工技术, 2019(6):134-137.

[4] Knill B. Application of Manufacturing Execution Systems [J]. Industry Week,1996, 245(9):8-10.

[5] 饶运清,李培根等.制造执行系统的现状与发展趋势 [J].机械 科学与技术, 2002,21(6):1011-1016.

[6] 俞鹏超,杨建军.工作流在MES中的设计与实现[J].先进制造 技术, 2005,24(3):34-36.

[7] 周万坤,朱剑英.面向制造过程的工作流建模[J]. 机械科学 与技术, 2004, 23(2):230-233.

[8] Ed Barkmeyer,Nenno P,Feng S, et al. NIST Response to MES Request for Information [R]. NIST Response to RFI3: MES Models, 1999.

[9] MESA International. MES explained : A high level vision [M]. American: MESA International White Paper Number 6, 1997.

[10] 常智勇,赵杰, 莫蓉. 复杂产品装配执行过程数字化技术研 究[J]. 南京航空航天大学学报, 2009, 41(5): 564-569.

[11] 何非,李东波.基于BOM的可配置制造执行系统研究 [J].制造 自动化,2015, 37(1):23-28.

[12] 柳丹,郑禄,帖军.基于物联网的智能制造执行系统设计与实 现[J].软件导刊,2016,15(2):87-89.

[13] 李波,李辉,陈鹰. 可重构制造执行系统的研究 $[\mathrm{J}]$.机械科学与 技术,2006,25(6):721-724.

[14] 杨铁江.基于物联网的制造企业信息化智能网络[J].中国制 造业信息化,2011,40(17):1-5.

[15] 夏昕嵘,邵学梅,赵峰.基于MES的智能库管系统设计与实现 [J].电气应用, $2019, v .38(\mathrm{~S} 1): 133-137$. 
[16] 刘振宇,刘瑾,厉娜.智能车间MES产业技术 [J].中国科技信息, 2020, No.629(11):18-22.

[17] 游健平.生产车间生产管理信息系统设计与构想 $[\mathrm{J}]$.电脑知 识与技术,2020, v.16(30): 84-85, 90 .
[18] 韩溧.面向智能仓储的制造执行系统的设计与实现 $[D]$. 西安 电子科技大学, 2020 . 